\title{
Possibilities for Simulating Clothing in Motion on Person-Specific Avatars
}

\author{
Doudou ZHANG*, Sybille KRZYWINSKI, Yordan KYOSEV \\ TU Dresden, Institute of Textile Machinery and High Performance Material Technology, Germany
}

https://doi.org/10.15221/21.08

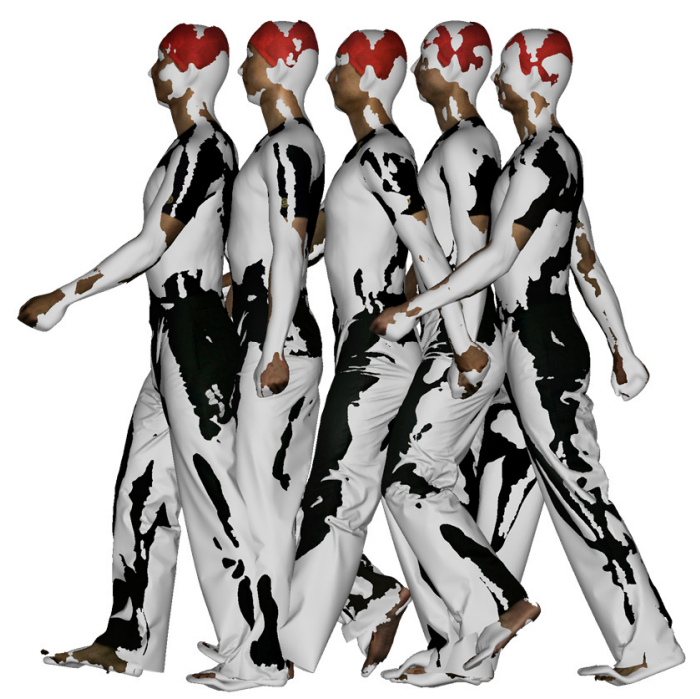

Fig.1. Comparison between real (black) and simulated (grey) clothing

\begin{abstract}
Efficient modeling of realistic clothing deformation remains a challenge. Our work presents an approach for numerical evaluation of simulated clothing in consideration of simulation errors. Our method considers the fabric properties and compares real clothing and virtual clothing. Our method generates a believable comparison between both in motion. We make it possible to do quantitative evaluation based on motion capture, not only visual evaluation based on picture and video.
\end{abstract}

Keywords: 4D scan, Clothing simulation, Animation

\section{Introduction}

For many years, the garment industry has been trying to shift product development more and more to the virtual level to save material and personnel costs and shorten product development times. For this purpose, avatars in standard sizes or personalized are elaborated, and simulation algorithms for virtual sewing of the garments are created. Furthermore, it is essential to correctly model the variety of textile materials in their deformation/drape behavior. The equipment technology required to determine the mechanical material parameters is increasingly being established in the textile and clothing industry. $3 \mathrm{D}$ garment simulation software allows the repetitive simulation of the nude body and the garment as desired in a still posture and motion [1]. To represent clothing in motion and thus analyze the esthetic appearance and the required functionality, moving avatars are necessary. In the context of this research study, the fit of a pair of trousers is to be virtually represented and analyzed based on three movements (walking, high knees, sitting/standing up). The material behavior plays an essential role in the wearing comfort. For this reason, the trousers will be made of a stretch-resistant material (fabric with a slight stretch) and a material with a high proportion of elastane. The relevant material parameters for deformation behavior simulation are determined and animated in the Clo3D software.

The skeletal structures used to animate the surface of the test person represent a simplification of the actual skeleton as regards the number of joints and bones. To check how close to reality the movement of person-specific data can be represented in this simplification, animations are presented as examples. The resulting data is used to simulate the constructed clothing in motion and to evaluate the wearing comfort based on the stress-strain behavior and the esthetic appearance during movement. This forms a basis for adapting the material selection to the functionality in the future without having to go through an extensive trial and error phase.

\footnotetext{
* doudou.zhang@tu-dresden.de; +49 (0) 351 479-4029-1; http://tu-dresden.de/mw/itm
} 
Furthermore, a geometric comparison of scan data of a test person in clothing and simulated data is carried out to determine how the material behavior can be reproduced in previous simulation solutions.

\section{State of the art}

So far, the studies to compare real garments and virtual simulation were mostly done on manikins in stationary postures using advanced 3D scanning and post-processing methods[2]-[5]. Luible [6] attempts to compare virtual and real garments in dynamic posture. The fit simulation is animated. A mesh must be attached to the virtual skeleton. The body animations are mainly obtained with motion tracking systems. The dynamic garment simulations on virtual manikins are more precise in fabric mechanics but not in body animation [6]. But the computation of the exact fabric mechanics is very time consuming.

The validation of $3 \mathrm{D}$ simulation software for visualizing the fit of garments was done only by qualitative comparison of photos and videos due to the poor posture and movement control. The nude and dressed bodies need to be in exactly the same position for superimposition, which is impossible with living subjects [1]. When living people are scanned, the major difficulty is the occurrence of scanning artifacts due to involuntary body movements and breathing during the scanning period and tissue compression and displacement by the garment. The principle of such evaluation is to align dressed, and undressed body scans made in the same body posture, which is hard to control in humans even when using a marking and supporting construction for the limbs and head [7].

Theoretically, it is possible to analyze the similarities between real and virtual garments during movement since some high-speed systems (Move 4D, Spain) capture up to 180 frames per second and enable the capture of $3 \mathrm{D}$ data in motion. Subsequently, the captured frames in motion can be transferred to post-processing to obtain the 3D animation.

Realistic simulation of garments on real humans is one of the fascinating areas of computer graphics and engineering. Over the past several years, mathematicians, physicians, and engineers have carried out considerable research to find algorithms to model clothes [8]. Some software programs, like LECTRA Modaris [9], offer for example the option to fit the garment on an avatar in a static position, with the sole option to lower the arms for a more "relaxed" pose. Others like Clo3D [10] and Browzwear [11] offer the option to add animations to your avatar in simulation mode. This means that the avatar can move in specific ways. Browzwear even has the option to have the avatar assume sports-related postures. This offers the option to look at the fit and strain map during one of these animations, enhancing the fit assessment.

However, modeling real humans for virtual garments remains a challenge; many efforts have been directed at this problem [12]-[16]. For example, a pregnant body [17] was created to design clothing for pregnant women. Another work presents a method for analyzing clothing deformation during motion and detecting areas with less deformation, where wires and tapes can be placed on the garment [18]. The available avatar in most fit simulation software uses Linear Blend Skinning (LBS), attaching the surface of a mesh to an underlying skeletal structure. The problem with LBS is that it often produces unnatural results. Data-driven methods attempt to solve this problem by using large amounts of data, often in the form of 3D body scans or performance capture data. While these techniques are powerful interpolation tools, they have a limited extrapolation capability, which can only be compensated by collecting more and more data [14]. Another approach is to create a physics-based anatomical model that produces realistic anatomical movements of muscle, fat, and skin. Unfortunately, designing anatomically realistic humans is labor-intensive, even for experienced digital artists who use professional modeling and simulation tools [14].

Researchers have tried to capture human motion by using scanning techniques [19]-[21]. Fit testing of garments in motion is an important issue for workwear and sportswear since garments should not limit the range of motion. Therefore, dynamic anthropometry is an important research topic. However, it is not known how body geometry changes during dynamic movements [19].

\section{Experiment description}

Figure 2 below explains the steps that were followed in order to achieve the research study objective. 


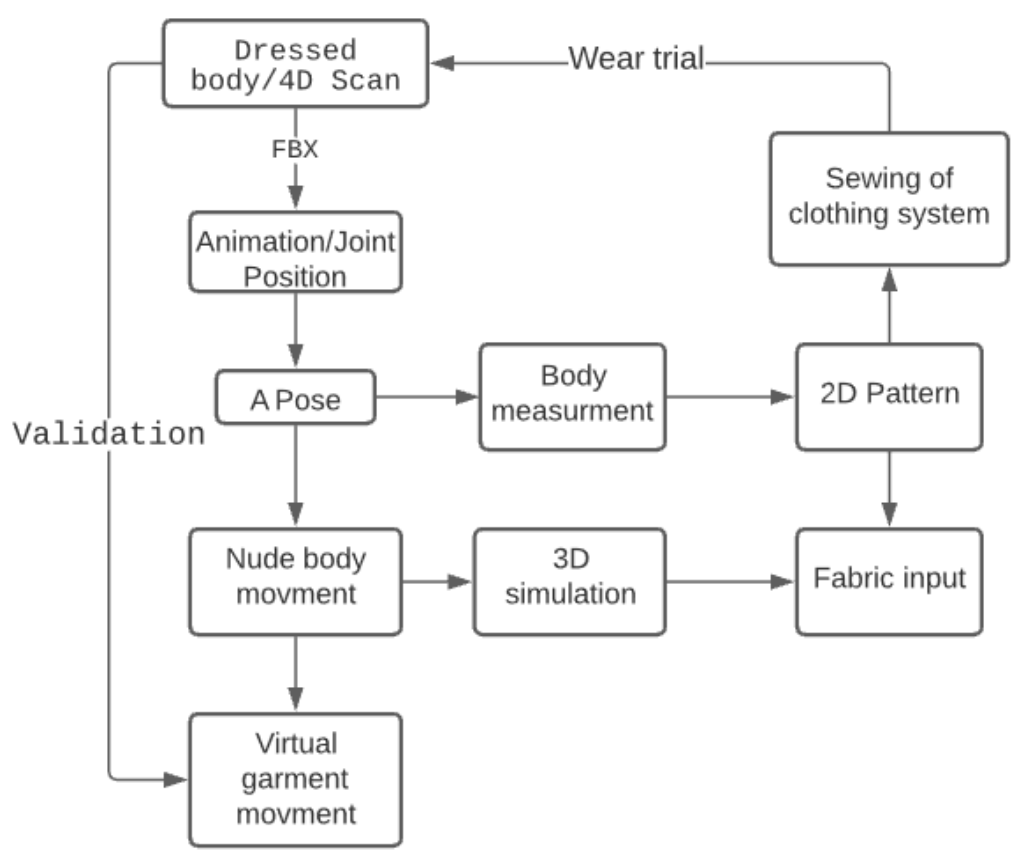

Fig.2. Workflow

- Development of 2D pattern cuts - the 2D CAD software Grafis was used for producing the pattern cuts according to the size of the selected test person and the design of the clothing system,

- Fabrics characterization - the physical, mechanical characteristics required for further simulation work were measured.

- Comparison of real and virtual fit (static simulation)

- 4D scanning of the test person - the selected test person was scanned by a 4D scanner (Move4D)

\subsection{Development and construction of custom pants}

The 2D patterns of basic pants for the test person were developed using the 2D CAD software Grafis [22]. The body measurements of the test person were imported into Grafis and used as basic body measurements for the construction of basic blocks. Figure 3 shows the basic construction of pants.

\begin{tabular}{|l|c|}
\hline Body height (cm) & 183 \\
\hline Waist circumference (cm) & 74 \\
\hline Ankle circumference (cm) & 26.5 \\
\hline Below knee circumference (cm) & 37 \\
\hline Thigh circumference (cm) & 55.5 \\
\hline Hip circumference (cm) & 94 \\
\hline Inseam (cm) & 88 \\
\hline Waist height (cm) & 117 \\
\hline Knee height (cm) & 62 \\
\hline
\end{tabular}

Fig.3. The basic construction of pants

The 2D patterns of the pants were plotted, and pattern cuts were prepared according to the selected textile fabric. These pattern cuts were sewn together to create a pair of pants for scanning the dressed test person. 


\subsection{Fabrics properties}

The ultimate goal of clothing simulation is to create a simulated garment that is nearly indistinguishable from the real clothing being modeled. If the simulated fabric behaves exactly like the real fabric, the simulation of the clothing can be started successful. In order to achieve realistic modeling of a specific garment, a reliable technique is needed that can accurately extract the physical properties of the fabric. Currently, the final feel of simulated clothing is varied by adjusting the stiffness (in bending, stretching, and shearing), damping, air drag, and mass [23].

Consequentially, the look of the clothing is determined by the material properties. While changing the stiffness and bending coefficients would be easily possible, the physical interpretation of this is unclear, and the results would be difficult to predict. Exploring the effect of different material properties would be an interesting application.

The selection of the textile fabrics to develop pants was made by considering the fabrics that are usually used for daily activities. The selected textile fabrics were characterized according to the requirements of fit simulation. Some 3D software developers use currently available standard measurement techniques to obtain the physical and mechanical properties needed to simulate garments. Others develop their own instruments, in both cases to facilitate the needs of user to digitize the mechanical and physical behavior of their own material. The latter is a key requirement for achieving a true virtual fitting based on measured properties [24]. In total, one warp, one weft, and one bias fabric specimen, each of $22 \mathrm{~cm}$ by $3 \mathrm{~cm}$, are required in Clo3D to measure the weight, thickness, bending, and tensile properties. All measurements need to be read from the rulers and/or devices, then written into a table, and entered into the fabric emulator of the software [25]. An overview of the fabric properties can be seen in Table 1.

Table 1. Overview of the fabric properties

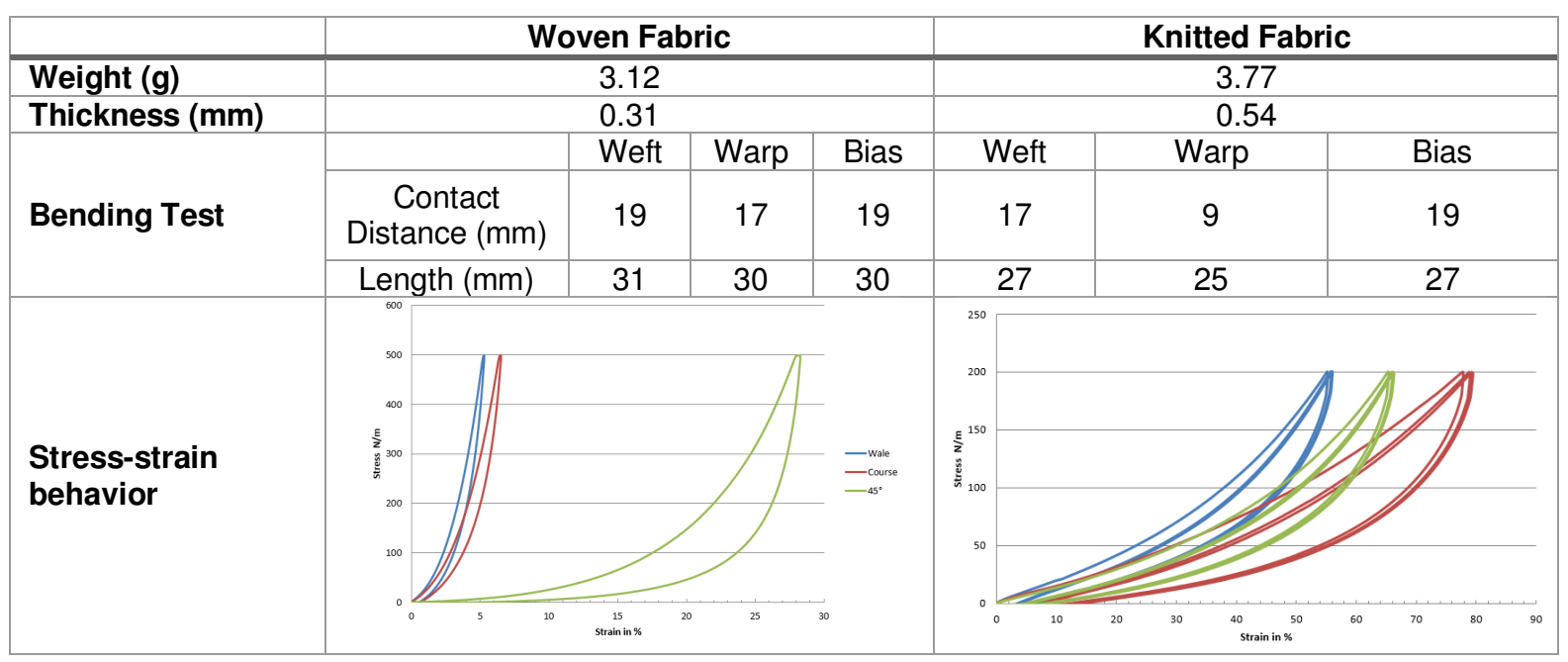

\subsection{Comparison of real and virtual comfort performance (static simulation)}

The 2D patterns of pants developed in Grafis and exported as AAMA/DXF file format were imported into Clo3D. A physics-based simulation using Clo3D returns a mesh that imitates similar material properties as our scan and contains 49,530 vertices. 
Table 2. Comparison between real and virtual clothing

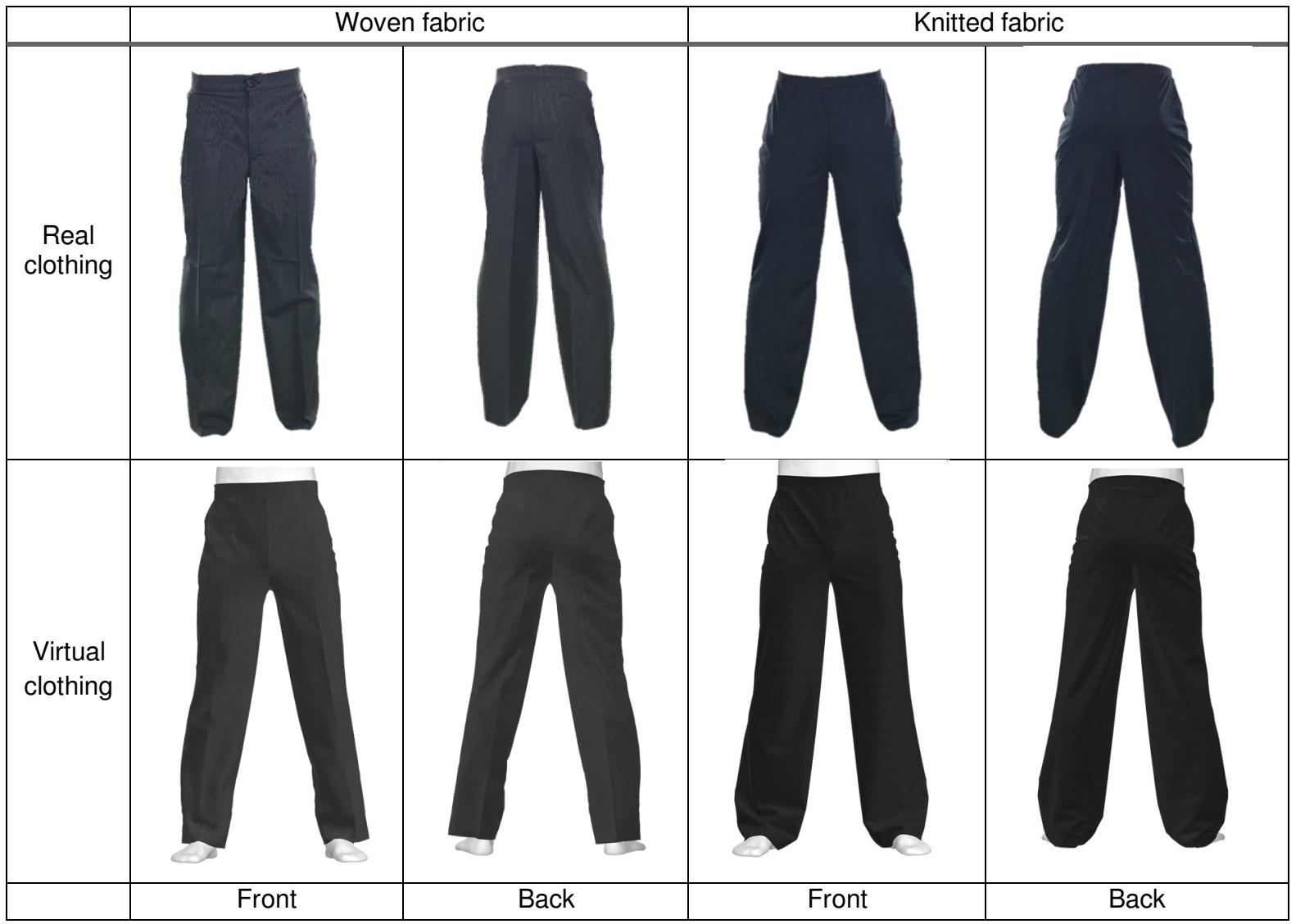

\subsection{D scanner (Move 4D)}

To study and simulate 3D shapes in motion, IBV (Spain, Valencia) provided us with a unique 4D scanner that can capture data at speeds up to $180 \mathrm{fps}$ [26]. It consists of a set of synchronized 4D scanning modules (12 modules) and processing software. This facility enables us to capture human bodies in motion at a spatial resolution of $1 \mathrm{~mm}$. The processing software makes the point cloud useful, like a watertight mesh. It is fully automatic for bodies in tight-fitting garments and does not require any interactive land marking or revision [27].

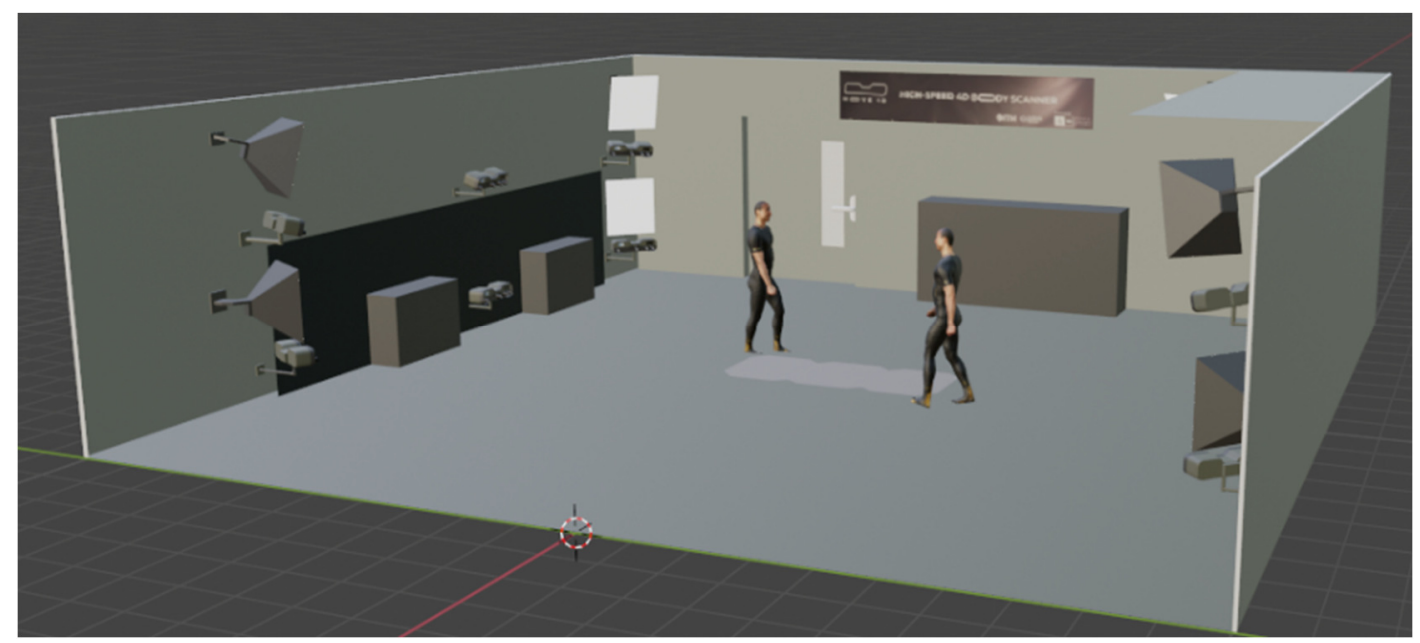

Fig.2. 3D\&4D Scan Lab 


\subsection{Capture of 4D-scans of a participant in motion}

In the present study, the lower body was chosen for analyzing possibilities to simulate clothing in motion on person-specific avatars. Table 2 shows the recordings of selected movements in different situations. The fit of a pair of pants is to be virtually represented and analyzed based on three movements (walking, high knee, sit to standup). We captured 60 frames (15 frames/second) for each movement.

We scanned the participant with $4 \mathrm{D}$ scanner twice in close-fitting leggings and loose-fitting pants. The scan files were converted to the FBX and OBJ formats.

Table 2. Virtual human model in motion

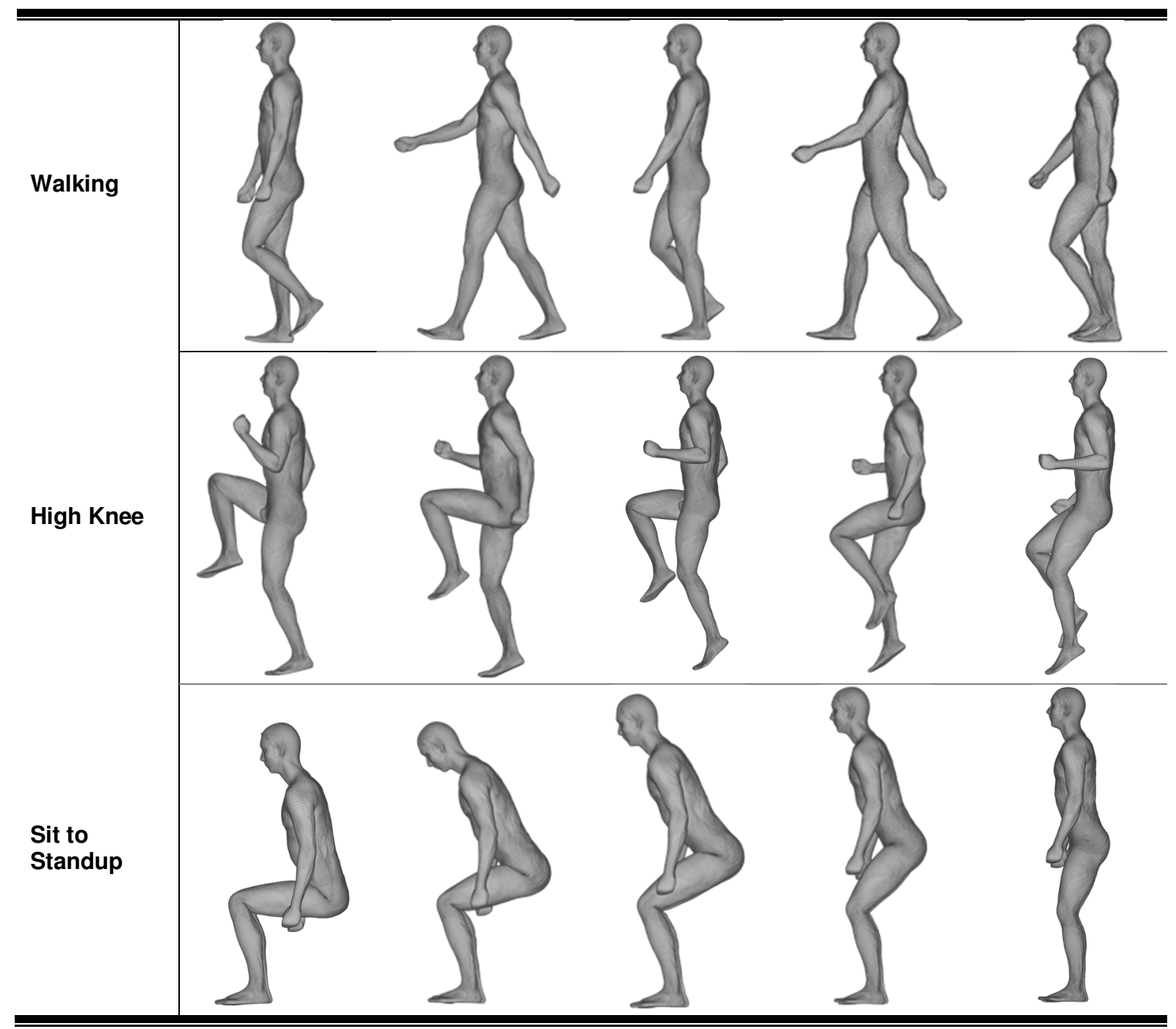

\subsection{Preparation of the 3D virtual bodies for fit simulation in motion}

To solve the problem addressed in the literature [1], the scans of nude and dressed bodies must have exactly the same position for the overlay, which is not possible for living persons. We have developed an algorithm to transfer the joint position of dressed body scans to the undressed body. When living people are scanned, the main difficulties are scan artifacts caused by involuntary body movements and breathing during the scan, as well as possible tissue compression and displacement due to clothing. Our research aims to align the dressed, and undressed body scans in the same body posture, which is hard to control in humans even when using a marking and supporting construction for the limbs and head (see Figure 3. a). The main functions of the developed algorithm are loading the FBX file content, creating a motion dictionary (holds all translation and rotation values for every joint and every frame), writing motion dictionary values, and creating keyframes. We have an A-pose with a rigid skeleton (Figure 3. b: undressed; Figure 3. c: dressed) from the 4D scanner, a surface mesh, and a set of 
skinning weights that determine the surface deforms according to the pose of the avatar. Since the model has the same skeleton structure, whether undressed and dressed, we can transfer the coordinates of the joints from model $\mathrm{c}$ to model $\mathrm{b}$. We construct a kinematic clothing deformation model that will serve as the reference. In the end, we can align the simulated and scanned clothing in motion (see Figure 3.d).

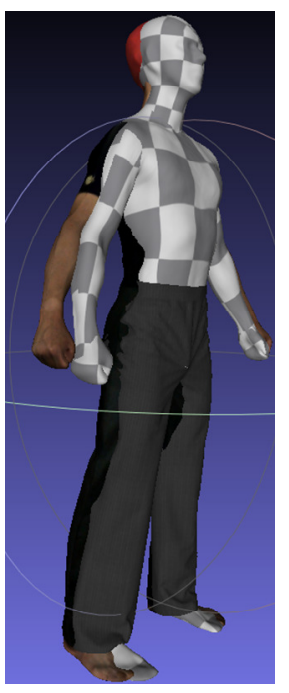

a

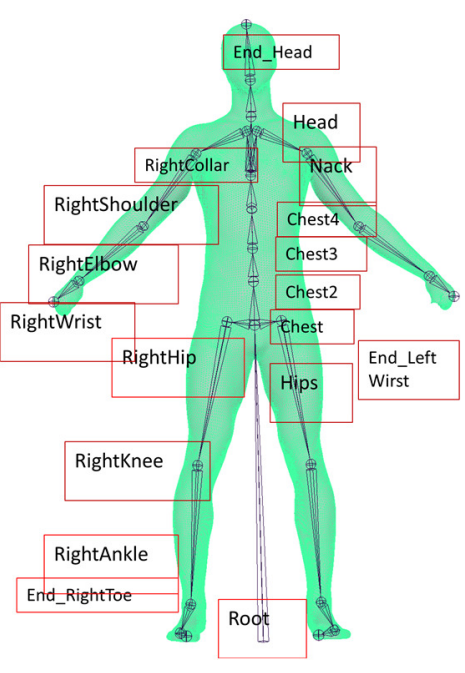

b

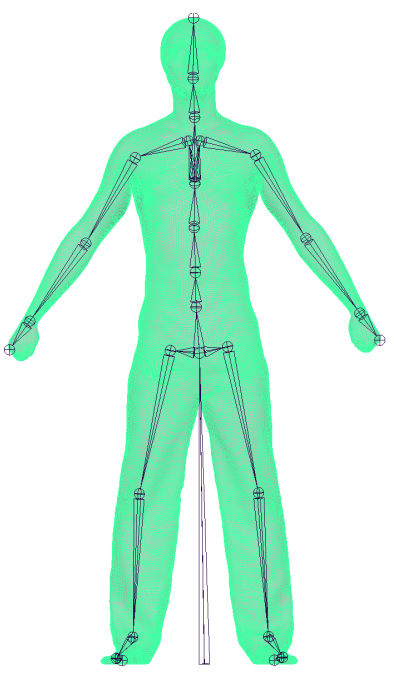

C

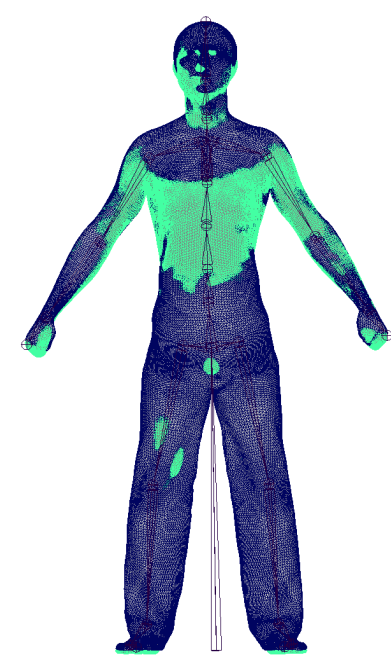

d

Fig.3.a: Different posture; b: undressed body with skeleton; c: dressed body with the same skeleton structure; Alignment between dressed and undressed body scan.

\section{Results}

The motion of walking was selected as an example to simulate the clothing in motion. We also scanned the dressed test person. We observed that the scan could contain holes and the registration of the 4D model body was not robust to describe the shape of the pants, and so we could not achieve an accurate "clothing level". We attempt to achieve the highest quality scan right out of the gates that requires almost no post-scanning manipulation. As we do not intend to clean or edit the scan aggressively, any missing geometry would be an issue.

In a high-quality simulation, the surface of the scan should be represented by the surface of the simulated clothing. For each vertex on the scan, we find its distance to the reference template (scan). Figure 4 (woven fabric) and Figure 6(knitted fabric) show distance maps indicating the per-vertex alignment error to better understand the spatial distribution of alignment errors. It shows that in all frames the aligning most of the vertices is good. Where they differ, most is in the lower part of the pants. Figure 5 (woven fabric) and figure 7 (knitted fabric) further describe the error between real and virtual clothing in motion.\$ 


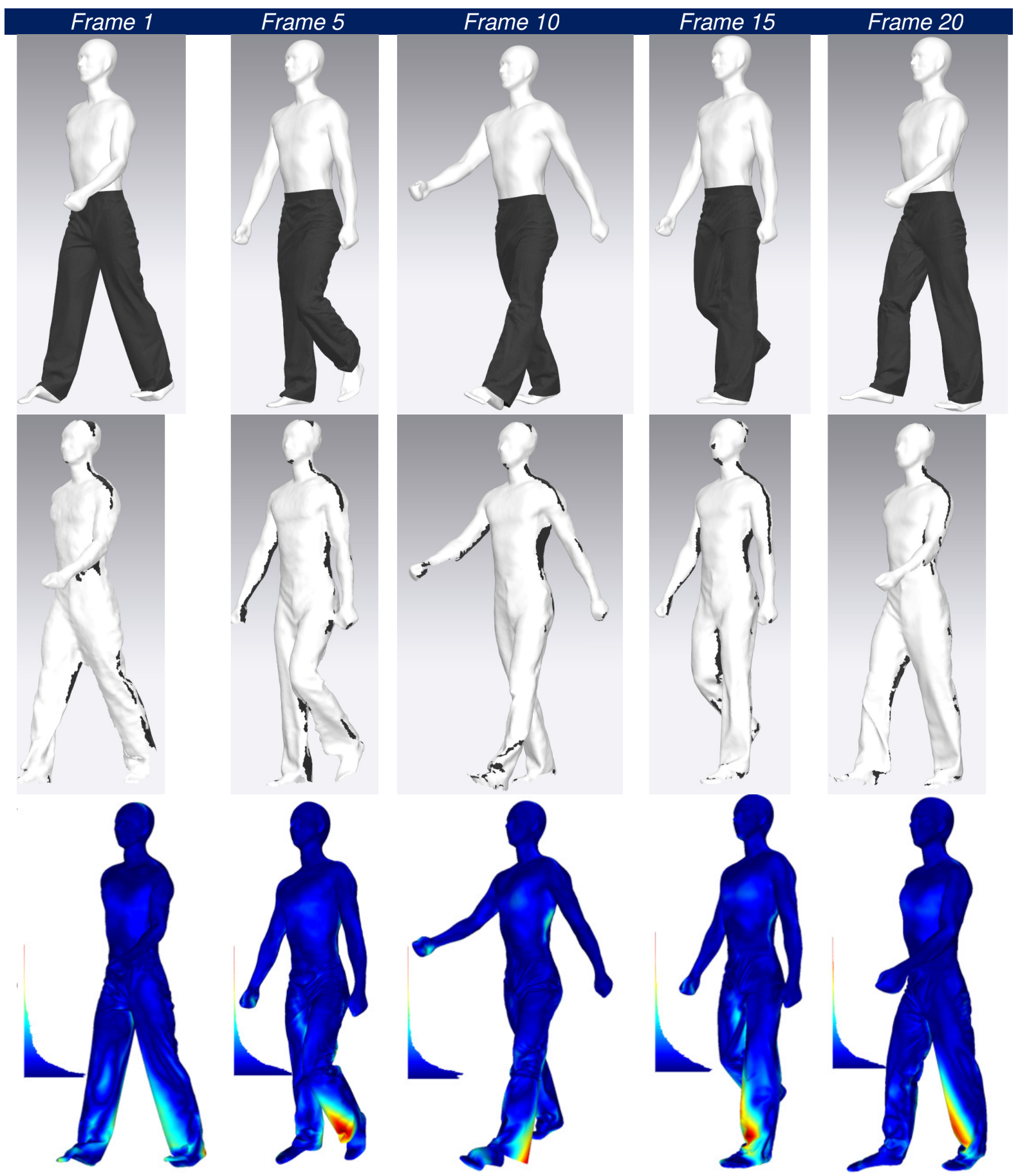

Fig.4. Woven fabric; Top: Simulated pants in motion; Middle: Scanned pants in motion; Bottom: Comparison
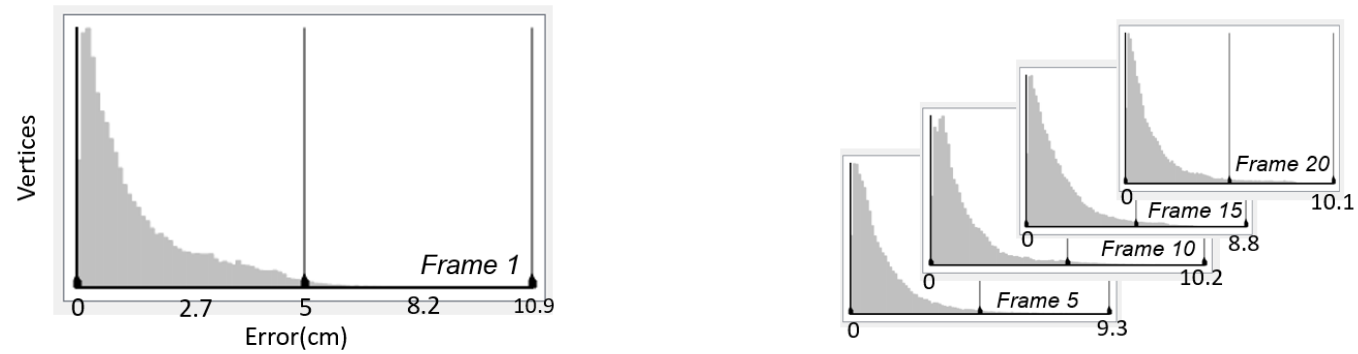

Fig.5. Woven fabric; Distance from scan to simulated clothing 
Table 3 (woven fabric) and Table 4 (knitted fabric) shows the minimum, maximum and mean distance from scans to simulated clothing.

Table 3. Woven fabric; Mean mesh distances are computed by averaging errors over the simulated clothing

\begin{tabular}{|l|c|c|c|c|c|}
\hline & Frame 1 & Frame 5 & Frame 10 & Frame 15 & Frame 20 \\
\hline Minimum (cm) & $\mathbf{0 . 0 0}$ & $\mathbf{0 . 0 0}$ & $\mathbf{0 . 0 0}$ & $\mathbf{0 . 0 0}$ & $\mathbf{0 . 0 0}$ \\
\hline Maximum (cm) & $\mathbf{1 0 . 9 0}$ & $\mathbf{9 . 3 0}$ & $\mathbf{1 0 . 2 4}$ & $\mathbf{8 . 7 8}$ & $\mathbf{1 0 . 1 1}$ \\
\hline Mean(cm) & $\mathbf{1 . 3 3}$ & $\mathbf{1 . 0 8}$ & $\mathbf{1 . 1 2}$ & $\mathbf{1 . 1 3}$ & $\mathbf{1 . 2 7}$ \\
\hline
\end{tabular}

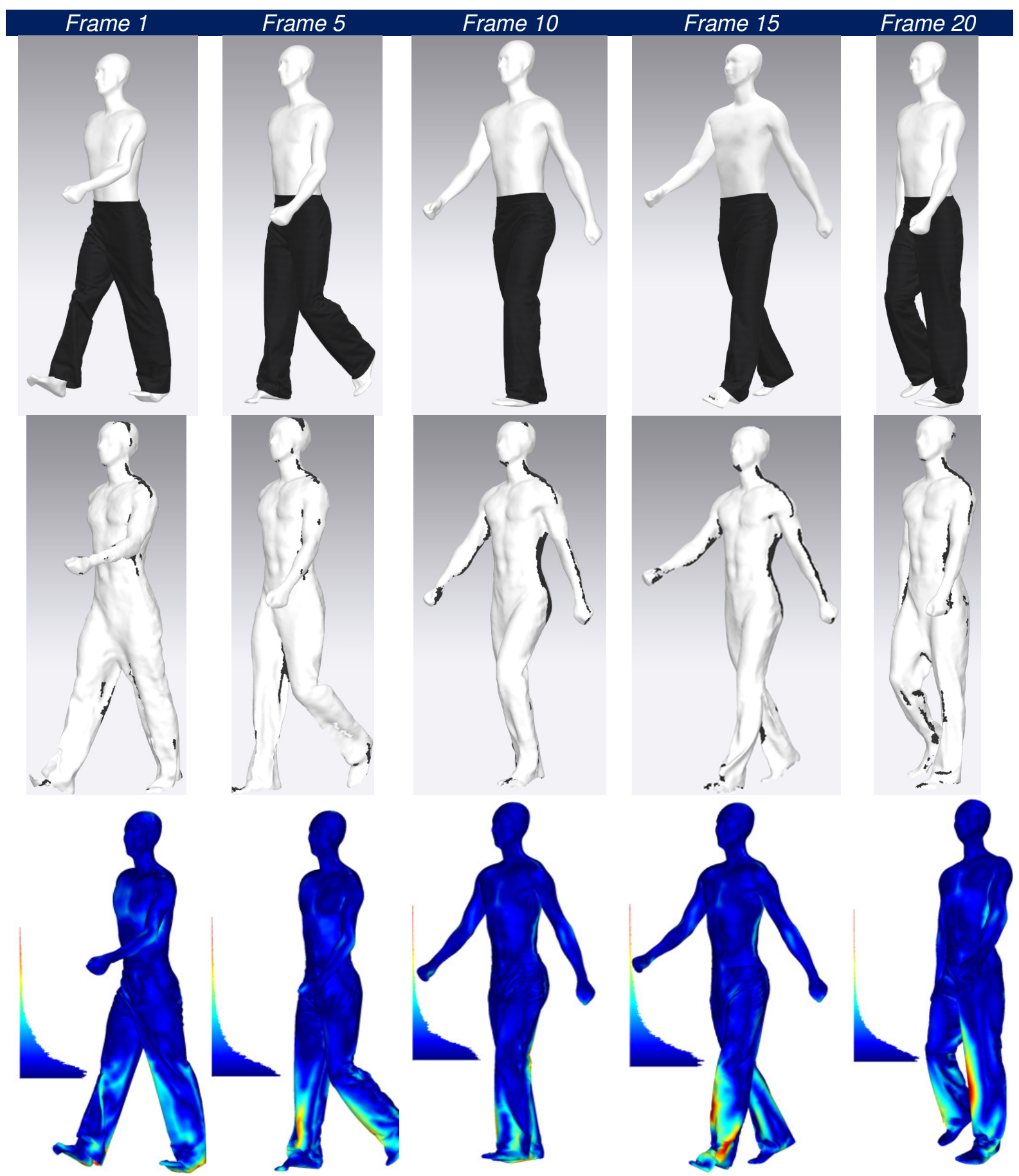

Fig.6. Knitted fabric; Top: Simulated pants in motion; Middle: Scanned pants in motion; Bottom: Comparison 
Table 4. Knitted fabric; Mean mesh distances are computed by averaging errors over the simulated clothing

\begin{tabular}{|l|c|c|c|c|c|}
\hline & Frame 1 & Frame 5 & Frame 10 & Frame 15 & Frame 20 \\
\hline Minimum $(\mathrm{cm})$ & $\mathbf{0 . 0 0}$ & $\mathbf{0 . 0 0}$ & $\mathbf{0 . 0 0}$ & $\mathbf{0 . 0 0}$ & $\mathbf{0 . 0 0}$ \\
\hline Maximum $(\mathrm{cm})$ & $\mathbf{8 . 4 8}$ & $\mathbf{8 . 4 2}$ & $\mathbf{8 . 3 5}$ & $\mathbf{7 . 2 9}$ & $\mathbf{7 . 5 1}$ \\
\hline Mean $(\mathrm{cm})$ & $\mathbf{1 . 3 0}$ & $\mathbf{1 . 8 5}$ & $\mathbf{1 . 7 1}$ & $\mathbf{1 . 0 6}$ & $\mathbf{1 . 0 0}$ \\
\hline
\end{tabular}
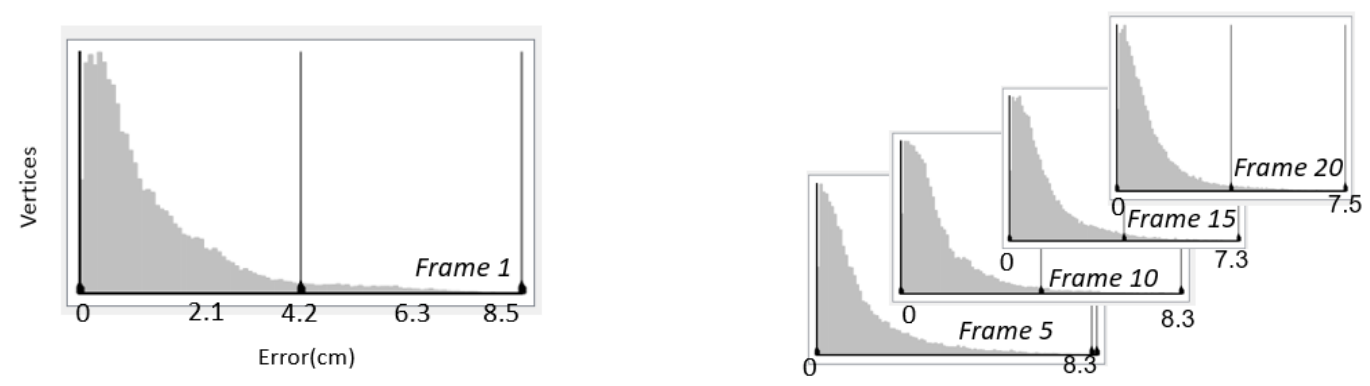

Fig.7. Knitted fabric; Distance from scan to simulated clothing

\section{Conclusions}

In this paper, we propose a new method for comparing simulation results and 4D scans in motion. We evaluated the accuracy of the simulation results based on the distance between the scan and the simulation.

We found the simulation of the garment does not satisfactorily reproduce the movement of the clothing. We observed significant errors in the lower part of the pants. The errors came from the fabric side, including fabric measurements, parameter derivation, and limitations of the simulation algorithm.

\section{Acknowledgment}

The research project of the Research Community Forschungskuratorium Textil e. V. is funded by the AiF within the program "Industrial Collective Research (IGF-21603 BR/1)" of the German Federal Ministry of Economic Affairs and Energy (BMWi) by a resolution of the German Bundestag.

\section{References}

[1] E. Mert u. a., „A validation methodology and application of 3D garment simulation software to determine the distribution of air layers in garments during walking“, Measurement, Bd. 117, S. 153-164, März 2018, doi: 10.1016/j.measurement.2017.11.042.

[2] Y. Y. Wu, P. Y. Mok, Y. L. Kwok, J. T. Fan, und J. H. Xin, „An investigation on the validity of 3D clothing simulation for garment fit evaluation", Juni 2016. Zugegriffen: Juli 06, 2020. [Online]. Verfügbar unter: http://ira.lib.polyu.edu.hk/handle/10397/52652

[3] M. Ernst, U. Detering-Koll, und D. Güntzel, „Investigation on Body Shaping Garments Using 3DBody Scanning Technology and 3D-Simulation Tools", gehalten auf der Proc. of 3rd Int. Conference on 3D Body Scanning Technologies, Lugano, Switzerland, Oct. 2012, Okt. 2012. doi: 10.15221/12.064.

[4] „Comparative Study of Real and Virtual Garments Appearance and Distance Ease | Semantic Scholar". https://www.semanticscholar.org/paper/Comparative-Study-of-Real-and-VirtualGarments-and-Lag\%C4\%97-Ancutien\%C4\%97/109ffdcc1d93d83d1d1e40ffac564cbdf0952474 (zugegriffen Aug. 04, 2021).

[5] „[PDF] An investigation on the validity of 3D clothing simulation for garment fit evaluation | Semantic Scholar". https://www.semanticscholar.org/paper/An-investigation-on-the-validity-of3D-clothing-for-Wu-Mok/7497045079fe4a4d2fd79a3951a6fd4afe6511ba (zugegriffen Aug. 04, 2021).

[6] C. Luible, „Study of mechanical properties in the simulation of 3D garments“, University of Geneva, 2008. doi: 10.13097/archive-ouverte/unige:688. 
[7] A. Psikuta, J. Frackiewicz-Kaczmarek, E. Mert, M.-A. Bueno, und R. M. Rossi, „Validation of a novel 3D scanning method for determination of the air gap in clothing", Measurement, Bd. 67, S. 61-70, Mai 2015, doi: 10.1016/j.measurement.2015.02.024.

[8] J. Power, „Fabric objective measurements for commercial 3D virtual garment simulation“, International Journal of Clothing Science and Technology, Nov. 2013, doi: 10.1108/IJCST-122012-0080.

[9] Modaris Expert: innovative 2D/3D-lösung für modellerstellung. https://www.lectra.com/de/produkte/modaris-expert (zugegriffen Juni 29, 2021).

[10] CLO | 3D Fashion Design Software. https://www.clo3d.com/ (zugegriffen Juni 29, 2021).

[11] Fashion Design Software", Browzwear. https://browzwear.com/ (zugegriffen Juni 29, 2021).

[12] M. Loper, N. Mahmood, J. Romero, G. Pons-Moll, und M. J. Black, „SMPL: A Skinned Multiperson Linear Model“, ACM Trans. Graph., Bd. 34, Nr. 6, S. 248:1-248:16, Okt. 2015, doi: 10.1145/2816795.2818013.

[13] F. Bogo, J. Romero, G. Pons-Moll, und M. J. Black, „Dynamic FAUST: Registering Human Bodies in Motion", in 2017 IEEE Conference on Computer Vision and Pattern Recognition (CVPR), Juli 2017, S. 5573-5582. doi: 10.1109/CVPR.2017.591.

[14] P. Kadleček, A.-E. Ichim, T. Liu, J. Křivánek, und L. Kavan, „Reconstructing Personalized Anatomical Models for Physics-based Body Animation“, ACM Trans. Graph., Bd. 35, Nr. 6, S. 213:1-213:13, Nov. 2016, doi: 10.1145/2980179.2982438.

[15] J. Jacobs, J. Barbic, E. Edwards, C. Doran, und A. van Straten, „How to build a human: practical physics-based character animation", in Proceedings of the 2016 Symposium on Digital Production, Anaheim, California, Juli 2016, S. 7-9. doi: 10.1145/2947688.2947698.

[16] S. Barth und E. Wendt, „Development of individual soft orthoses for the conservative treatment of neuro-orthopaedic diseases“, Proceedings, ADDITC, Dresden 2019.

[17] K. Cieśla, I. Frydrych, S. Krzywinski, und Y. Kyosev, „Design workflow for virtual design of clothing for pregnant women“, Communications in Development and Assembling of Textile Products, Bd. 1, Nr. 2, Art. Nr. 2, Dez. 2020, doi: 10.25367/cdatp.2020.1.p148-159.

[18] D. Zhang, S. Krzywinski, und Y. Kyosev, „Analysis of Clothing Deformation During Motion and its Application for the Design of Functional Clothing", gehalten auf der Proc. of 3DBODY.TECH 2020 - 11th Int. Conference and Exhibition on 3D Body Scanning and Processing Technologies, Online/Virtual, 17-18 Nov. 2020, Nov. 2020. doi: 10.15221/20.09.

[19] A. Klepser und S. Morlock, „4D Scanning - Dynamic View on Body Measurements“, cdatp, Bd. 1, Nr. 1, Art. Nr. 1, Sep. 2020, doi: 10.25367/cdatp.2020.1.p30-38.

[20] A. Klepser, S. Morlock, C. Loercher, und A. Schenk, „7 - Functional measurements and mobility restriction (from 3D to 4D scanning)", in Anthropometry, Apparel Sizing and Design (Second Edition), N. Zakaria und D. Gupta, Hrsg. Woodhead Publishing, 2020, S. 169-199. doi: 10.1016/B978-0-08-102604-5.00007-X.

[21] M. Mahnic, S. Petrak, und Z. Stjepanovič, Analysis of Tight Fit Clothing 3D Construction Based on Parametric and Scanned Body Models. 2016, S. 313. doi: 10.15221/16.302.

[22] „Home - GRAFIS Software - Dr. K. Friedrich GbR“. https://www.grafis.com/ (zugegriffen Juni 30, 2021).

[23] K.-J. Choi und H.-S. Ko, „Research problems in clothing simulation“, Computer-Aided Design, Bd. 37, Nr. 6, S. 585-592, Mai 2005, doi: 10.1016/j.cad.2004.11.002.

[24] „IEEE MFPVS - THE MEASUREMENT OF FABRIC PROPERTIES FOR VIRTUAL SIMULATION-A CRITICAL REVIEW | Engineering360". https://standards.globalspec.com/std/14229997/IEEE\%20MFPVS.

[25] CLO3D Members, CLO Fabric Kit 2.0. Zugegriffen: Aug. 05, 2021. [Online Video]. Verfügbar unter: https://www.youtube.com/watch?v=HA7HoK6_4Fk

[26] „Move 4D“, Instituto de Biomecánica. https://www.ibv.org/en/move-4d/ (zugegriffen Juni 30, 2021).

[27] „MOVE 4D: Accurate High-Speed 3D Body Models in Motion“. https://www.researchgate.net/publication/336721053_MOVE_4D_Accurate_High Speed_3D_Body_Models_in_Motion. 